\title{
COOPERATIVE SPATIAL MULTIPLEXING IN MULTI-HOP WIRELESS NETWORKS
}

\author{
Yimin Zhang, Genyuan Wang, and Moeness G. Amin \\ Wireless Communications and Positioning Lab \\ Center for Advanced Communications \\ Villanova University, Villanova, PA 19085, USA
}

\begin{abstract}
It is well known that a multiple-input-multiple-output (MIMO) system can provide spatial diversity gain as well as spatial multiplexing capability. The MIMO concept has been extended to cooperative wireless networks to form distributed MIMO systems using virtual antennas located at cooperating terminals. The primary interest of cooperative MIMO networks, however, has been focused on the cooperative diversity (C-DIV) approaches to achieve spatial diversity gain. Recent work proposed cooperative spatial multiplexing (C-SM) to simplify the transmit and receive processing requirement on the relay nodes while providing significant energy savings. So far $C$-SM has been only considered for singlehop relaying. In this paper, we propose the use of multihop relaying $C$-SM systems for transmit energy reduction and performance improvement.
\end{abstract}

\section{INTRODUCTION}

The multiple-input-multiple-output (MIMO) systems can provide spatial diversity gain as well as spatial multiplexing capabilities. The MIMO approaches have been extended to a cooperative wireless network, where antennas over different terminals (nodes) cooperative with each other to form a virtual (or distributed) array. In a wireless network, transmission of information through relaying can be energy efficient. The use of multiple relaying nodes allows high diversity gain and leads to improved robustness against channel impairments [1].

So far, the primary interest of cooperative network has been focused on the cooperative diversity (C-DIV) approach, which, as the name implies, is intended to achieve spatial diversity gain to combat multipath fading in wireless communication environments [2]-[5].

A different and more recent approach is cooperative spatial multiplexing (C-SM). In a C-SM system, the

This work is supported in part by the NSWCCD/ONR under contract No. N65540-05-C-0028 and NSF under grant No. EEC0203459 . source uses high constellation modulations, and each relay node in the first hop (referred to as multiplexing relay node hereafter) only transmits a sub-stream of the source information. In this way, the transmit and receive processing requirements on the relay nodes are simplified $[6,7]$. In addition, compared to C-DIV, the C-SM approaches provide significant savings in the transmit and receive energy. It is most effective in the high signal-to-noise ratio (SNR) regime when no channel coding is performed, while the efficiency in the low SNR regime can be improved with appropriate channel coding schemes. C-SM is particularly useful in sending high-rate data when each relay node can handle only low-rates due to highly limited resources in terms of energy, bandwidth, computation power, memory, and space [6].

In this paper, we extend C-SM to multi-hop wireless networks. It is shown that, by using multiple sets of relay nodes, employing the amplify-and-forward algorithm, the C-SM approach works well even without exploiting any channel coding. The use of the proposed multi-hop C-SM schemes reduces the total transmit energy, particularly when assume a high path loss exponent.

Unlike C-DIV signals which provide sufficient information to allow single node to decode, the data transmitted from the C-SM multiplexing nodes usually cannot be decoded at a single node in the following hops. Similar to the BLAST schemes in a co-located MIMO system, it requires an array of more co-located sensors than the number of multiplexing factor (i.e., the number of multiplexing nodes). Therefore, in a multi-hop relaying scheme adopting C-SM, it is appropriate for the relay nodes to exploit the amplifying-and-forward algorithm, rendering the processing at the relay nodes very simple. This is because the amplify-and-forward algorithm does not involve complicated processing, and the computationally extensive demultiplexing operations are performed at the destination receiver, which is often resource-abundant.

To simplify the problem statement and provide a clear presentation of the proposed approach, we assume 
that the channels experience flat fading and the relay nodes are perfectly synchronized. The extension of the proposed method to imperfectly synchronized wireless network can be realized by incorporating the methods developed in $[8,9]$.

\section{SYSTEM MODEL}

A block diagram of a multi-hop distributed MIMO system adopting the C-SM scheme is depicted in Fig. 1. The primary focus of this paper is the introduction of multiple hops in the C-SM relay process. Similar to [6], we make the following assumptions. (1) The source and relay nodes are each equipped with a single antenna due to constraints on the size of the terminals; (2) The destination receiver is not constrained in size and can host multiple antennas; (3) Practical considerations prevent the relay nodes from transmitting and receiving concurrently at the same physical channel. To clearly present the multi-hop C-SM concept in the limited space available, no channel coding is considered in this paper.

The spatial multiplexing procedure at the multiplexing nodes is similar to that introduced in [6]. The source broadcasts a $2^{\alpha N}$-ary symbol $\bar{x}$ representing $\alpha N$ bits of information. The symbol energy is $E_{s}$, i.e., $E\left[|\bar{x}|^{2}\right]=E_{s}$, at a rate of $1 / T$ symbols per second. The multiplexing nodes $R_{1}^{(1)}, R_{2}^{(1)}, \cdots, R_{N}^{(1)}$ spatially map this information into $2^{\alpha}$-ary symbols $\left\{x_{1}, x_{2}, \cdots, x_{N}\right\}$, where the superscript ${ }^{(i)}$ denotes the $i$ th relay hop. Then, the multiplexing relay node $R_{i}^{(1)}$ detects only $x_{i}$ and forwards its estimate $\hat{x}_{i}$ to the relays in the following hop with a symbol energy $E\left[\left|\hat{x}_{i}\right|^{2}\right]=E_{r}^{(1)}$, at a rate of $1 / T$ symbols per second. At this step, all multiplexing relay nodes forward their estimates simultaneously to other relay nodes over the same physical channel.

The received signal $y_{i}^{(1)}$ at the multiplexing relay node $R_{i}^{(1)}$ is given by

$$
y_{i}^{(1)}=h_{i, S}^{(1)} x+n_{i}^{(1)}, \quad i=1, \cdots, N,
$$

where $h_{i, S}^{(1)}$ is the channel gain between the source and relay $R_{i}^{(1)}$, and $n_{i}^{(1)}$ is additive complex Gaussian noise with mean zero and variance $N_{0} / 2$ per real dimension. We assume that $h_{i, S}^{(1)}$ is a complex Gaussian distributed random variable with mean zero and variance $E\left[\left|h_{i, S}^{(1)}\right|^{2}\right]=d_{i, S}^{-\nu}$, where $d_{i, S}$ is the distance between the source $S$ and the relay $R_{i}^{(1)}$, and $\nu$ is the path loss exponent, typically $2 \leq \nu \leq 5$.

The signal transmitted from multiplexing relay node $R_{i}^{(1)}$ is

$$
x_{k}^{(1)}=g_{i}^{(1)} \hat{x}_{i}
$$

where $\hat{x}_{i}$ is the $2^{\alpha}$-ary symbol carrying the estimated information of $x_{i}, g_{i}^{(1)}$ is the transmit signal amplitude of node $R_{i}^{(1)}$. The received signal $y_{k}^{(2)}$ at the relay node $R_{k}^{(2)}$ of the next relay hop is given by

$$
\begin{aligned}
y_{k}^{(2)} & =\sum_{i=1}^{N} h_{k, i}^{(2)} x_{i}^{(1)}+n_{k}^{(2)} \\
& =\sum_{i=1}^{N} h_{k, i}^{(2)} g_{i}^{(1)} \hat{x}_{i}+n_{k}^{(2)}, \quad k=1, \cdots,\left(N^{*}\right)^{(2)}
\end{aligned}
$$

where $h_{k, i}^{(2)}$ is the channel gain between the $i$ th node in the first hop and the $k$ th node in the second hop, and $n_{k}^{(2)}$ is defined similar to $n_{k}^{(1)} .\left(N^{*}\right)^{(i)}$ denotes the number of nodes in the $i$ th relay hop, and we may abbreviate it as $N^{*}$ where there is no confusion. $h_{k, i}^{(2)}$ are assumed to be mutually uncorrelated and complex Gaussian distributed with mean zero and variance $E\left[\left|h_{k, i}^{(2)}\right|^{2}\right]=\left[d_{k, i}^{(2)}\right]^{-\nu}$, where $d_{k, i}^{(2)}$ is the distance between nodes $R_{i}^{(1)}$ and $R_{k}^{(2)}$.

In matrix form, equation (3) can be expressed as

$$
\mathbf{y}^{(2)}=\mathbf{H}^{(2)} \mathbf{G}^{(1)} \hat{\mathbf{x}}+\mathbf{n}^{(2)},
$$

where $\hat{\mathbf{x}}=\left[\hat{x}_{1}, \cdots, \hat{x}_{N}\right]^{T}, \mathbf{y}^{(i)}=\left[y_{1}^{(i)}, \cdots, y_{N^{*}}^{(i)}\right]^{T}, \mathbf{n}^{(i)}=$ $\left[n_{1}^{(i)}, \cdots, n_{N^{*}}^{(i)}\right]^{T},[\mathbf{H}]_{k, i}^{(2)}=h_{k, i}^{(2)}$, and $\mathbf{G}^{(i)}=\operatorname{diag}\left[g_{1}^{(i)}\right.$, $\left.\cdots, g_{N^{*}}^{(i)}\right]$. We use superscript ${ }^{T}$ to denote matrix or vector transpose.

\section{DISTRIBUTED MULTI-HOP RELAYING}

\subsection{Relay Procedures}

The signal transmitted from the $N$ multiplexing relay nodes is similar to that in a co-located V-BLAST system. Therefore, without redundancy introduced in the source signal by channel coding, a single relay node in the relay hops (other than the multiplexing relay hop) generally cannot decode the information it receives, making the use of decode-and-forward type of algorithms difficult to apply. As we show below, however, it is possible to perform multi-hop relays using the amplify-and-forward algorithm in the following relay stages. In this case, it is desirable that the number of relay nodes (more precisely, the rank of distributed MIMO relay channels) in each relay hop is equal to or larger than $N$.

The transmit signal from relay node $R_{k}^{(2)}$ is expressed as

$$
x_{k}^{(2)}=g_{i}^{(2)} \frac{y_{i}^{(2)}}{\left|y_{i}^{(2)}\right|}=\tilde{g}_{i}^{(2)} y_{i}^{(2)} \text {, }
$$

where $g_{i}^{(2)}$ is the transmit power at the node, and $\tilde{g}_{i}^{(2)}=$ $g_{i}^{(2)} /\left|y_{i}^{(2)}\right|$ is the amplification gain. Because this relay 


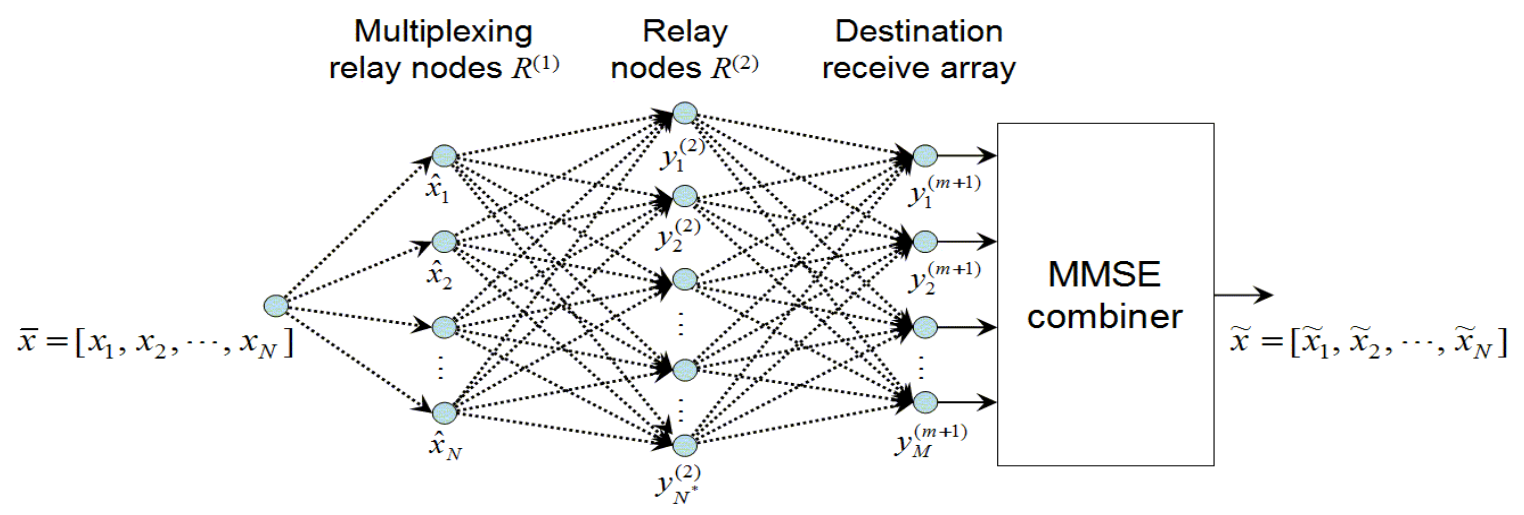

Figure 1: Multi-hop relaying system under consideration.

stage uses the amplify-and-forward algorithm, part of the transmit energy will be used to send receiver noise. As the result, the transmit energy used to deliver useful information is affected by the receive SNR at the relay nodes.

The received signal vector at the following relay hop or at the destination antenna array is expressed as

$$
\begin{aligned}
\mathbf{y}^{(3)}= & \mathbf{H}^{(3)} \mathbf{x}^{(2)}+\mathbf{n}^{(3)} \\
= & \mathbf{H}^{(3)} \tilde{\mathbf{G}}^{(2)} \mathbf{y}^{(2)}+\mathbf{n}^{(3)} \\
= & \mathbf{H}^{(3)} \tilde{\mathbf{G}}^{(2)} \mathbf{H}^{(2)} \mathbf{G}^{(1)} \hat{\mathbf{x}} \\
& +\left[\mathbf{H}^{(3)} \tilde{\mathbf{G}}^{(2)} \mathbf{n}^{(2)}+\mathbf{n}^{(3)}\right] .
\end{aligned}
$$

Therefore, $\mathbf{H}^{(3)} \tilde{\mathbf{G}}^{(2)} \mathbf{H}^{(2)} \mathbf{G}^{(1)}$ acts as the combined channels between the multiplexing relay nodes $R_{i}^{(1)}$ and relay hops $R_{i}^{(3)}$, whereas the noise over different relay hops are accumulated as $\mathbf{H}^{(3)} \tilde{\mathbf{G}}^{(2)} \mathbf{n}^{(2)}+\mathbf{n}^{(3)}$. This discussion can be obviously extended to higher relay orders.

\subsection{Destination Processing}

After $m$ hops of relays, the information is delivered at the destination, which consists of $M$ co-located array sensors. It is assumed that $M \geq N$. The received signal vector at the destination array is expressed as

$$
\mathbf{y}^{(m+1)}=\prod_{l=1}^{m} \mathbf{H}^{(l+1)} \tilde{\mathbf{G}}^{(l)} \mathbf{y}^{(1)}+\tilde{\mathbf{n}}^{(m+1)},
$$

where $\mathbf{H}^{(m+1)}$ is the channel matrix between the nodes at the last relay hop and the destination array antennas, and $\tilde{\mathbf{n}}^{(m+1)}=\mathbf{H}^{(m+1)} \tilde{\mathbf{G}}^{(m)} \tilde{\mathbf{n}}^{(m)}+\mathbf{n}^{(m+1)}$ is the equivalent noise vector accumulated over all the relay stages. We used $\tilde{\mathbf{G}}^{(1)}=\mathbf{G}^{(1)}$ for convenience. Note that the noise vector $\mathbf{n}^{(m+1)}$ at destination receiver usually has variances different from those of the relay nodes.

The detection is similar to those methods developed for V-BLAST $[10,6]$. Such a receiver typically involves signal ordering, nulling, and feedback decision.

\subsection{Remarks}

From the above discussion, the equivalent channel matrix over the all $m$ hops of relay is $\tilde{\mathbf{H}}=\prod_{l=1}^{m} \mathbf{H}^{(l+1)}$ $\cdot \mathbf{G}^{(l)}$. Therefore, the rank of $\tilde{\mathbf{H}}$ is determined by the minimum rank of $\mathbf{H}^{(l)}$ and $\mathbf{G}^{(l)}$. Practically, $\mathbf{G}^{(l)}$ are full rank (i.e., $\operatorname{rank}\left[\mathbf{G}^{(l)}\right]=\left(N^{*}\right)^{(l)}$ for respective $l$, where $l=1, \cdots, m$, whereas the rank of $\mathbf{H}^{(l)}$ satisfies $\operatorname{rank}\left[\mathbf{H}^{(l)}\right] \leq \min \left[\left(N^{*}\right)^{(l-1)},\left(N^{*}\right)^{(l)}, l=2, \cdots, m+1\right.$. To construct an efficient multi-hop C-SM relay system, therefore, it is desirable to have $\left(N^{*}\right)^{(l)} \geq N, l=$ $2, \cdots, m$. As we will show by numerical results, increasing the number of relay nodes in each hop increases the diversity gain. Thus, by keeping the total transmit energy unchanged in each relay hop, the use of more relay nodes improves the transmission performance.

\section{SIMULATION RESULTS}

We present two sets of simulation results. The first set compares the BER performance of a multi-hop CSM system to that of a corresponding single-hop C-SM system. In the second set of simulations, the relationship between BER and the number of relay nodes is examined.

Consider a scenario that the distance between the source and the destination is $100 \mathrm{~m}$. The source node transmits $2^{8}$-ary QAM signals to four multiplexing relay nodes $(N=4)$, which are located from the source node at a distance of $5 \mathrm{~m}$. Each of these relay nodes employs QPSK modulation. The resulting spectral efficiency is $4 \mathrm{bps} / \mathrm{Hz}$. The BER performance of a singlehop C-SM relay system and that of a three-hop system are compared. The path loss exponential is set to $\nu=4$. Both systems use the same total transmit energy. For the three-hop system, the distance between two node belong to adjacent relay hops is the same for all hops, i.e., $d=(100-5) / 3=31.67 \mathrm{~m}$. The transmit energy is equally distributed to the three relay hops. The bit error rate (BER) results are depicted in Fig. 2 . The $E_{b} / N_{0}$ is evaluated at a destination receiver when all the energy is transmitted from a single source 


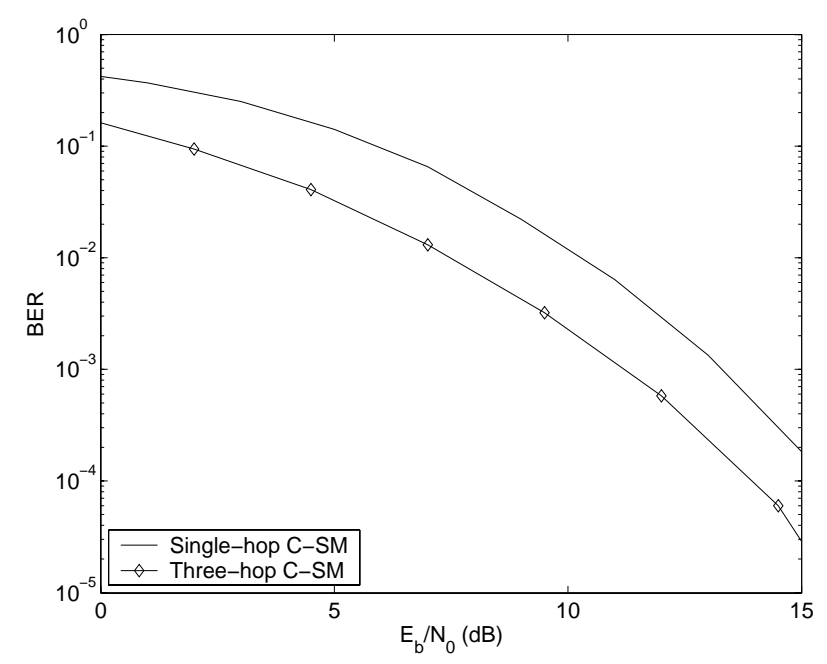

Figure 2: BER versus receive $E_{b} / N_{0}$ for single- and three-hop C-SM systems. $N=\left(N^{*}\right)^{(2)}=\left(N^{*}\right)^{(3)}=$ $M=4$.

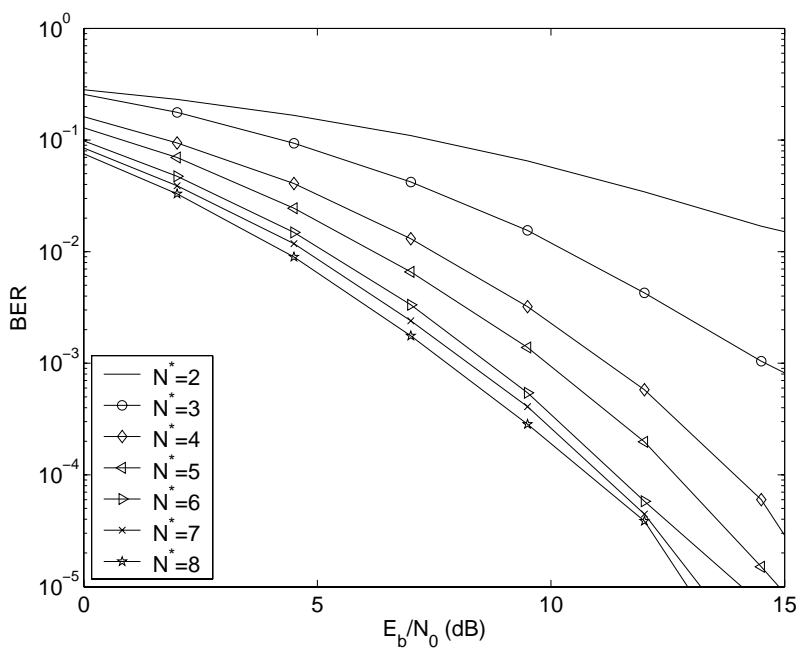

Figure 3: BER versus number of relay nodes $\left(N^{*}\right)^{(2)}=$ $\left(N^{*}\right)^{(3)}=N^{*}$ in a three-hop C-SM system. $N=M=$ 4 .

node without relays. It is evident from the plots that, by using multi-hop relays, the BER is improved and, equivalently, the transmit energy can be saved at the source and relay nodes.

In the second set of simulations, we focus on the three-hop relay system discussed above, with the number of multiplexing relay nodes and that of the destination receive antennas fixed to 4 (i.e., $N=M=4$ ). We let the number of relay nodes in the two intermediate relay hops, $\left(N^{*}\right)^{(2)}=\left(N^{*}\right)^{(3)}=N^{*}$, change from 2 to 8 , while the total transmit energy in each hop remains unchanged. The results are depicted in Fig. 3. It is evident that, the performance is poor when $N^{*}<N$. As the number of relay nodes increases, the BER performance is improved.

\section{CONCLUSIONS}

In this paper, we have proposed the use of multi-hop relay scheme in a cooperative spatial multiplexing (CSM) system. We have shown that, while the signal decoding at each relay node in the second and later relay hops is generally impractical, the exploitation of relatively simple amplify-and-forward algorithm allows energy-efficient signal relaying, leaving the computationally expensive detection operation of the spatially multiplexed information exclusively to the destination receiver. Simulation results have shown significant performance improvement and energy savings by using intermediate relay hops in a C-SM system.

\section{REFERENCES}

[1] A. Sendonaris, E. Erkip, and B. Aazhang, "User cooperative diversity - Part I and Part II," IEEE Trans. Commun., vol. 51, no. 11, pp. 1927-1948, Nov. 2003.

[2] J. N. Laneman and G. W. Wornell, "Distributed SpaceTime Coded Protocols for Exploiting Cooperative Diversity in Wireless Networks", IEEE Trans. Inform. Theory, vol. 49, no. 10, pp. 2415-2425, Oct. 2003.

[3] G. Wang, Y. Zhang, and M. G. Amin, "Space-time cooperation diversity using high-rate codes," Int. Symp. Antennas Propagat., Sendai, Japan, Aug. 2004.

[4] G. Wang, Y. Zhang, and M. Amin, "Cooperation diversity using differential distributed space-time codes," Joint Conf. of Asia-Pacific Conf. on Commun. and Int. Symp. on Multi-Dimensional Mobile Commun., Beijing, China, Aug. 2004.

[5] Y. Hua, Y. Mei, and Y. Chang, "Parallel wireless mobile relays with space-time modulations," IEEE Workshop on Statistical Signal Processing, St. Louis, MO, Sept. 2003.

[6] S. W. Kim and R. Cherukuri, "Cooperative spatial multiplexing for high-rate wireless communications," IEEE Workshop in Signal Processing Advances in Wireless Communications, New York, pp. 181-185, June 2005.

[7] S. W. Kim and R. S. Cherukuri, "Adaptive forwarding and coding in cooperative spatial multiplexing system," IEEE Vehicle Technology Conference, Dallas, TX, Sept. 2005.

[8] X. Li, "Space-time coded multi-transmission among distributed transmitters without perfect synchronization," IEEE Signal Processing Letters, vol. 11, no. 12, pp. 948951, Dec. 2004.

[9] Y. Zhang, G. Wang, and M. G. Amin, "Imperfectly synchronized cooperative network using distributed spacefrequency coding," IEEE Vehicular Technology Conference, Dallas, TX, Sept. 2005.

[10] G. J. Foschini, "Layered space-time architecture for wireless communication in a fading environment when using multi-element antennas," Bell Labs Techn. J., pp. 41-59, 1996. 